\title{
Negative Control of RpoS Synthesis by the sRNA ReaL in Pseudomonas aeruginosa
}

\author{
Hue Thi Bach Nguyen 1t, David Romero A. 1t, Fabian Amman², \\ Theresa Sorger-Domenigg ${ }^{1}$, Muralidhar Tata ${ }^{1}$, Elisabeth Sonnleitner ${ }^{1}$ and Udo Bläsi ${ }^{1 *}$ \\ ${ }^{1}$ Department of Microbiology, Immunobiology and Genetics, Max F. Perutz Laboratories, Center of Molecular Biology, \\ University of Vienna - Vienna Biocenter, Vienna, Austria, ${ }^{2}$ Institute of Theoretical Chemistry, University of Vienna, Vienna, \\ Austria
}

Pseudomonas aeruginosa (Pae) is an opportunistic human pathogen, able to resist host defense mechanisms and antibiotic treatment. In Pae, the master regulator of stress responses RpoS $\left(\sigma^{S}\right)$ is involved in the regulation of quorum sensing and several virulence genes. Here, we report that the sRNA ReaL translationally silences rpoS mRNA, which results in a decrease of the RpoS levels. Our studies indicated that

OPEN ACCESS

Edited by:

Satoshi Tsuneda,

Waseda University, Japan

Reviewed by:

Silvia Ferrara,

Università degli Studi di Milano, Italy

Franz Narberhaus,

Ruhr-Universität Bochum, Germany

*Correspondence:

Udo Bläsi

Udo.Blaesi@univie.ac.at

${ }^{\dagger}$ These authors have contributed equally to this work

Specialty section:

This article was submitted to Microbial Physiology and Metabolism,

a section of the journa

Frontiers in Microbiology

Received: 12 July 2018 Accepted: 28 September 2018 Published: 29 October 2018

Citation:

Thi Bach Nguyen $H$, Romero A D, Amman $F$, Sorger-Domenigg $T$,

Tata M, Sonnleitner E and Bläsi U (2018) Negative Control of RpoS Synthesis by the SRNA ReaL

in Pseudomonas aeruginosa.

Front. Microbiol. 9:2488.

doi: 10.3389/fmicb.2018.02488 ReaL base-pairs with the Shine-Dalgarno region of rpoS mRNA. These studies are underlined by a highly similar transcription profile of a rpoS deletion mutant and a reaL over-expressing strain.

Keywords: Pseudomonas aeruginosa, ReaL, RpoS, translational silencing, small RNA

\section{INTRODUCTION}

Pseudomonas aeruginosa (Pae) is an opportunistic human pathogen that causes severe infections in immunocompromised individuals, burn patients, and patients suffering from cystic fibrosis. Owing to its ability to form biofilms, Pae can resist host defense mechanisms and antibiotic treatment (Costerton et al., 1999). Many Pae virulence factors are controlled by numerous two component systems, extra-cytoplasmic sigma factors, and by the four inter-linked quorum sensing (QS) systems Las, Rhl, Pqs, and Iqs (Van Delden and Iglewski, 1998; Lee and Zhang, 2015).

Different sigma factors control different subsets of genes, enabling bacteria to adapt to environmental changes (Wosten, 1998). While expression of housekeeping genes is regulated by $\sigma^{70}$, Pae encodes a number of additional sigma factors (Martínez-Bueno et al., 2002), one of which is $\operatorname{RpoS}\left(\sigma^{S}\right)$, considered to be the master regulator of stress responses. RpoS is predominantly expressed during stationary growth (Fujita et al., 1994) and under other stress conditions, including low $\mathrm{pH}$, heat shock, oxidative stress, and increased osmolarity (Jorgensen et al., 1999; Cochran et al., 2000; Venturi, 2003; Schuster et al., 2004; Dong and Schellhorn, 2010). As RpoS controls the synthesis of quorum-sensing (QS) dependent response regulators in Pae, RpoS governs the expression of a large subset of genes including functions required for the synthesis of virulence factors such as pyocyanin, exotoxin A, LasA and LasB elastases, and exoenzyme S (Suh et al., 1999; Sonnleitner et al., 2003; Hogardt et al., 2004).

Bacterial sRNAs play a major role in post-transcriptional regulation. Their expression is usually induced by environmental cues and leads to a fast response and adaptation to stressors and/or habitats (Hoe et al., 2013). In general sRNAs modulate gene expression in a positive- or negative manner by base-pairing with their target mRNAs, which often requires the RNA chaperone Hfq (Storz et al., 2011). In addition, RNAs can mediate gene expression by sequestration of 
regulatory proteins, as exemplified by the Pae RNAs CrcZ (Sonnleitner and Bläsi, 2014) and RsmW/RsmY/RsmZ (Lapouge et al., 2008; Miller et al., 2016). While numerous candidate sRNAs have been identified in several Pae strains (Livny et al., 2006; Sonnleitner et al., 2008; Sonnleitner and Haas, 2011; Ferrara et al., 2012; Gómez-Lozano et al., 2012; Wurtzel et al., 2012; Caldelari et al., 2013) only a few, including the sRNAs PhrS (Sonnleitner et al., 2011), NrsZ (Wenner et al., 2014), PrrF1-2 (Reinhart et al., 2015), ErsA (Ferrara et al., 2015), PesA (Ferrara et al., 2017), sr0161, and Sr006 (Zhang et al., 2017), have been functionally characterized.

In E. coli, the expression of rpoS is predominantly regulated post-transcriptionally. The E. coli $5^{\prime}$ untranslated region (UTR) of rpoS mRNA contains a stem-loop structure that occludes the ribosome binding site (RBS) (Majdalani et al., 1998). Binding of sRNAs to this region disrupts the secondary structure and results in enhanced rpoS mRNA translation, which is accompanied by stabilization of the mRNA (Majdalani et al., 1998; Majdalani et al., 2002; McCullen et al., 2010). In E. coli, the three Hfq-binding sRNAs, DsrA, RprA, and ArcZ, are known to activate rpoS mRNA translation through this mechanism (Majdalani et al., 1998; Mika and Hengge, 2014), whereas OxyS RNA represses RpoS synthesis by a mechanism that is not fully understood (Zhang et al., 1998). Similarly, as observed for OxyS RNA in E. coli, ectopic expression of the RpoS-dependent sRNA RgsA decreased RpoS synthesis in a Hfq-dependent manner during exponential growth phase in Pae (Lu et al., 2018). However, the latter authors did not obtain evidence for base-pairing between rpoS mRNA and RgsA. In addition, the physiological implications for these findings remain unclear as RgsA did not impact on RpoS synthesis during stationary growth, when the increase in RpoS levels results in increased rgs A expression (Lu et al., 2018).

Carloni et al. (2017) characterized the Pae PA14 sRNA SPA0084 (Ferrara et al., 2012) as a QS regulator that is upregulated during stationary growth. They termed the sRNA ReaL for 'regulator of 2-alkyl-4(1H)-quinolone'. The reaL gene, which is highly conserved among 13 Pae strains, is located in Pae strain $\mathrm{PAO} 1$ in the intergenic region between the two open reading frames PA3535 and PA3536 (Carloni et al., 2017; Supplementary Figure S1A). The analysis of the promoter region of reaL revealed a consensus motif for $\mathrm{s}^{70}$ and RpoS, respectively (Carloni et al., 2017). The RpoS dependency was supported by the finding that ReaL synthesis did not increase during stationary phase in a PA14 rpoS deletion mutant (Carloni et al., 2017). It was further shown that ReaL acts as a link between the Las and PQS systems. Carloni et al. (2017) reported that LasR negatively regulates reaL transcription and that ReaL interacts with and positively regulates pqs C translation in a Hfq-independent manner, which finally results in increased PQS production and in the establishment of PQS dependent virulence traits such as pyocyanin production, biofilm formation, and swarming motility. These findings could be reconciled with an attenuated and a hyper-virulent phenotype of a reaL deletion strain and a reaL overexpressing strain, respectively.

The Pae sRNA SPA0084/ReaL has been independently identified in our laboratory as PaeIII (Sorger-Domenigg, 2010). A comparative RNA $_{\text {Seq }}$ based transcriptome analysis of a reaL overproducing PAO1 strain and a PAO1 reaL deletion strain revealed striking similarities with the expression profile obtained with a PAO1 rpoS deletion strain (Schuster et al., 2004). Using sRNA target identification by ligation (Han et al., 2016) and $r p o S:: l a c Z$ translational reporter gene fusions, we provide evidence that ReaL directly represses rpoS mRNA translation through base-pairing with a sub-sequence encompassing the Shine and Dalgarno sequence of the latter. Hence, we identify ReaL as the first bacterial sRNA that translationally silences $r p o S$ mRNA through a base-pairing mechanism.

\section{MATERIALS AND METHODS}

\section{Bacterial Strains and Growth Conditions}

Strains and plasmids used in this study are listed in Supplementary Table S1. Unless indicated otherwise, the cultures were grown aerobically at $37^{\circ} \mathrm{C}$ in Luria-Bertani (LB; Miller, 1972) broth supplemented with appropriate antibiotics.

The PAO1 $\Delta$ reaL strain was constructed through homologous recombination as previously described (Ye et al., 1995). Briefly, the upstream and downstream sequences of reaL, spanning the regions -655 to -26 and +62 to +666 with regard to the transcriptional start site of reaL, were amplified from PAO1 chromosomal DNA by PCR using primer combinations V34/W34 and Q32/R32 (Supplementary Table S2), respectively. The 629 nt upstream PCR product was cloned into plasmid pSUP202 using the restriction sites PstI and PvuI to generate pSUP202-ReaLup. The 604 nt downstream PCR product, containing the reaL rho-independent terminator sequence, was then cloned into plasmid pSUP202-ReaLup using the restriction sites $P v u I$ and EcoRI, resulting in the generation of plasmid pSUP202-ReaLko. This plasmid (suicide vector) was transformed into the E. coli strain S17-1 and then transferred by conjugation to PAO1. The plasmid was chromosomally integrated by homologous recombination in the presence of tetracycline. The excision of the vector by a second crossover event was achieved by enrichment for tetracycline-sensitive cells (Ye et al., 1995). The chromosomal deletion of reaL was verified by DNA sequencing and Northern-blot analysis.

\section{Construction of Plasmids}

The plasmids for over-expression of reaL and the reaL variant harboring a deletion spanning $n t+11$ to +18 with respect to its transcriptional start site were constructed as follows. Full length reaL as well as the rea $L_{\Delta 11-18}$ variant were amplified from PAO1 chromosomal DNA by PCR using primer pairs Y138/Z138 and E163/Z138 (Supplementary Table S2), respectively. The PCR fragments were then cloned into the XbaI, and PstI sites of plasmid pKH6. In the resulting plasmids, pKH6-ReaL and pKH6ReaL $_{\Delta 11-18}$, reaL and rea $L_{\Delta 11-18}$ are under the transcriptional control of an arabinose-inducible $\mathrm{P}_{\mathrm{BAD}}$ promoter.

A translational $r p o S:: l a c Z$ fusion under the transcriptional control of the rpoS promoter was constructed as follows. The rpos promoter and the first 56 rpos codons were amplified by PCR using oligonucleotides A52 and B52 (Supplementary Table S2), which contained a BamHI and a PstI restriction site, 
respectively. The PCR product was cloned into the corresponding restriction sites of plasmid pME6014 to generate plasmid pME6014-RpoS. This PCR product was also cloned into the corresponding restriction sites of plasmid pME6016 to generate plasmid pME6016-RpoS, harboring a transcriptional rpoS-lacZ reporter gene.

\section{RNA Isolation}

Total RNA was isolated using the hot phenol method as described by Leoni et al. (1996). Briefly, the cells were harvested by centrifugation. The pellet was resuspended in $50 \mu \mathrm{l}$ DEPC treated $\mathrm{ddH}_{2} \mathrm{O}$ and mixed with pre-heated $\left(65^{\circ} \mathrm{C}\right) 250 \mu \mathrm{l}$ lysis buffer and $500 \mu \mathrm{l}$ phenol ( $\mathrm{pH} 5.5$ ) and vigorously vortexed. After centrifugation ( $5 \mathrm{~min}$ at $16,000 \mathrm{~g}$ ), the aqueous phase was extracted with an equal volume of phenol/chloroform (1:1), re-centrifuged, and then extracted with an equal volume of chloroform. The RNA was precipitated over-night at $-20^{\circ} \mathrm{C}$, by addition of 0.1 volume of $3 \mathrm{M} \mathrm{Na}$-acetate $(\mathrm{pH} 5.5)$ and 2.5 volumes of $96 \%$ ethanol. Removal of DNA was achieved by multiple rounds of TURBO DNase I (ThermoFisher) treatment followed by phenol chloroform extraction and $\mathrm{Na}$ acetate/ethanol precipitation.

\section{Mapping of the ReaL Termini}

Simultaneous mapping of the $5^{\prime}$ and $3^{\prime}$ ends of ReaL by RACE (Rapid Amplification of cDNA Ends) using circularized RNAs was performed as previously described (Toledo-Arana et al., 2009). Briefly, $6 \mu \mathrm{g}$ total RNA were treated with DNase I (Roche) followed by phenol/chloroform extraction. The RNA was divided into two aliquots, one of which was treated with tobacco acid pyrophosphatase, (TAP) (Epicentre Biotechnologies) as specified by the manufacturer. After TAP treatment, the RNA was extracted with acid-phenol/chloroform followed by ethanol precipitation. Serial dilutions (from 500 to $0.5 \mathrm{ng}$ ) of the TAP+ and TAP- RNAs were prepared. Each dilution was ligated with $40 \mathrm{U}$ T4 RNA ligase I (Fermentas) in the presence of $1 \times$ RNA ligase buffer in a total volume of $25 \mu \mathrm{l}$ at $37^{\circ} \mathrm{C}$ for $1 \mathrm{~h}$. The RNA was again extracted with acidphenol/chloroform and precipitated with ethanol. The RNAs were resuspended in $10 \mu \mathrm{l}$ RNase-free water. The subsequent RTPCR reactions were performed with the oligonucleotides B53 and C53 (Supplementary Table S2) and the SuperScript One-Step RT-PCR kit (Invitrogen). DNA fragments of the expected size present in the TAP+ reactions were gel extracted, purified, and then cloned into the pGEM-T Easy Vector System (Promega). Five plasmid clones were sequenced and the sequences were compared to the PAO1 genome to localize the $5^{\prime}$ - and $3^{\prime}$-ends of ReaL.

\section{RNA-Sequencing}

PAO1 $\Delta$ reaL(pKH6) and PAO1 $\Delta r e a L(\mathrm{pKH} 6-\mathrm{ReaL})$ were grown in $\mathrm{LB}$ medium supplemented with $50 \mu \mathrm{g} / \mathrm{ml}$ gentamycin until they reached an $\mathrm{OD}_{600}$ of 2.5. ReaL synthesis was induced with L-arabinose (final concentration $0.2 \%$ ). After 30 min, total RNA was isolated from three biological replicates of either strain as described above. Ribosomal RNA was depleted with the Ribo-Zero rRNA Removal Kit (Illumina). Then, libraries were prepared using the NEBNext Ultra Directional RNA Library Prep Kit (Illumina) and sequenced using the Illumina HiSeq 2000, $100 \mathrm{bp}$ single end read platform at the Vienna Biocentre Core Facilities. ${ }^{1}$ After removal of the adaptors, the sequences were quality trimmed with trimmomatic using default parameters (Bolger et al., 2014). The resulting sequences were then mapped onto the PAO1 reference genome (NC_002516.2) using Segemehl (Hoffmann et al., 2009, 2014) with default parameters. To allow sequence visualization in the UCSC Genome Browser (Chan et al., 2012), the ViennaNGS toolbox (Wolfinger et al., 2015) was used. For differential gene expression analysis, reads were counted using BEDtools (Quinlan and Hall, 2010) and analyzed using the DESeq2 package (Love et al., 2014). All RNAs with a $\log _{2}$-fold change greater than \pm 1.5 and a multiple testing adjusted p-value below 0.05 were considered to be differentially abundant. The raw sequencing data were deposited in the European Nucleotide Archive (ENA) as a study under the accession number PRJEB28696.

\section{Northern-Blot Analysis}

The abundance of ReaL was determined by Northern blotting using $10 \mu \mathrm{g}$ total RNA. The RNA samples were denatured for $5 \mathrm{~min}$ at $65^{\circ} \mathrm{C}$ in loading buffer containing $50 \%$ formamide, separated on $8 \%$ polyacrylamide/8 $\mathrm{M}$ urea gels, and then transferred to nylon membranes by electroblotting. The RNAs were cross-linked to the membrane by exposure to UV light. The membranes were hybridized with gene-specific ${ }^{32} \mathrm{P}$-endlabeled oligonucleotides (ReaL: W28; 5S rRNA: I26), and the hybridization signals were visualized using a PhosphorImager (Molecular Dynamics).

\section{Determination of the Steady State Levels and Half-Life of rpoS mRNA by RT-qPCR in the Presence and Absence of ReaL}

PAO1 $\Delta r e a L(\mathrm{pKH} 6)$ and $\mathrm{PAO} 1 \Delta$ reaL $(\mathrm{pKH} 6-\mathrm{ReaL})$ were grown in LB medium supplemented with $50 \mu \mathrm{g} / \mathrm{ml}$ gentamycin until they reached an $\mathrm{OD}_{600}$ of 2.5. ReaL synthesis was induced with L-arabinose (final concentration $0.2 \%$ ). After $30 \min \left(\mathrm{t}_{0}\right)$, the first sample was withdrawn and rifampicin was added to a final concentration of $250 \mu \mathrm{g} / \mathrm{ml}$. Additional samples were then taken after 1,2, 5, and $7.5 \mathrm{~min}$, respectively. Then, total RNA was isolated from either sample as described above and DNase I treated. cDNA was synthesized from $2 \mu \mathrm{g}$ of DNA-free total RNA using random hexamer primers (Promega) and AMV reverse transcriptase (Promega) according to the instructions of the manufacturer. For qPCR, 100 ng cDNA was used as template from either sample. The qPCR was performed with rpoS specific primers $\mathrm{J} 98$ and $\mathrm{K} 98$. The transcript levels of the rpoD gene obtained with the primers Q117 and R117 were used for normalization of the $t_{0}$ samples as described by Lee et al. (2012). The real-time PCR mixture containing $5 \times$ HOT FIREPol EvaGreen qPCR Mix (Medibena), $100 \mathrm{ng}$ cDNA, and $250 \mathrm{nM}$ of each primer was placed in a Real-time PCR cycler (Eppendorf Mastercycler), and the reaction was started at $95^{\circ} \mathrm{C}$ for $5 \mathrm{~min}$,

${ }^{1}$ https://www.vbcf.ac.at/home/ 
followed by 40 cycles of $20 \mathrm{~s}$ at $95^{\circ} \mathrm{C}, 20 \mathrm{~s}$ at $55^{\circ} \mathrm{C}$, and $20 \mathrm{~s}$ at $72^{\circ} \mathrm{C}$. For all reactions including the DNA standards and the negative control (no template), two biological replicates and three technical replicates each were performed. The fluorescence was measured at the last step of each cycle. After 40 cycles, a melting curve analysis was performed by raising the temperature from $45^{\circ} \mathrm{C}$ to $95^{\circ} \mathrm{C}$ every $15 \mathrm{~s}$, and by measuring the fluorescence at each cycle. The melting curve analysis yielded a single peak in the melting curve. Changes in the rpoS levels were estimated as previously described (Pfaffl, 2001).

\section{Western-Blot Analysis}

The cells were cultured in $\mathrm{LB}$ broth until they reached an $\mathrm{OD}_{600}$ of 1.0. Then, ReaL synthesis was induced with L-arabinose $(0.2 \%$ final concentration), and the cells were cultured for another $30 \mathrm{~min}$. Aliquots containing cells from a total $\mathrm{OD}_{600}$ of 2 were harvested by centrifugation at $15,500 \mathrm{~g}$ for $2 \mathrm{~min}$, resuspended in $100 \mu \mathrm{l} \mathrm{Laemmli}$ buffer, and lysed by heating to $95^{\circ} \mathrm{C}$ for $5 \mathrm{~min}$. The cell lysates were then centrifuged at $26,000 \mathrm{~g}$ for $25 \mathrm{~min}$ and the pellets were discarded. A $10-\mu \mathrm{l}$ aliquot of each sample was separated on a $10 \%$ SDS-polyacrylamide gel, and the proteins were transferred to a nitrocellulose membrane (Amersham Protran, GE Healthcare Life Sciences) by electroblotting. The membranes were incubated for $1 \mathrm{~h}$ at room temperature in TBST [25 mM Tris ( $\mathrm{pH} 7.4$ ), $150 \mathrm{mM} \mathrm{NaCl}$, $0.05 \%$ Tween-20] containing $2.5 \%$ non-fat dry milk followed by washing in TBST. After washing, the membranes were probed at $4^{\circ} \mathrm{C}$ overnight with anti-RpoS antibodies. Probing with antiribosomal protein S1 antibodies served as a loading control. The membranes were washed in TBST and incubated with a goat anti-rabbit IgG horseradish peroxidase-linked antibody (Cell Signaling Technology, Europe) for $1 \mathrm{~h}$ at room temperature. Finally, the membranes were washed with TBST, and the proteins were detected with the enhanced chemiluminescence detection kit (Thermo Fisher Scientific, Inc.). Protein levels were calculated using the ImageLab work-suite (Bio-rad) and normalized against ribosomal protein S1 (internal control).

\section{Modified GRIL-Seq}

To corroborate the ReaL-rpos interaction, the GRIL-seq approach (Han et al., 2016) was employed. Briefly, the strains PAO1 $\Delta$ reaL (pKH-ReaL; pKH13-t4rnl1) and PAO1 $\Delta$ reaL (pKH6; pKH13-t4rnl1) were cultured in $15 \mathrm{ml} \mathrm{LB}$ broth supplemented with $50 \mu \mathrm{g} / \mathrm{ml}$ gentamicin and $250 \mu \mathrm{g} / \mathrm{ml}$ carbenicillin until they reached an $\mathrm{OD}_{600}$ of 1.8. T4 RNA ligase gene expression was induced with IPTG to a final concentration of $1 \mathrm{mM}$. After $1 \mathrm{~h}$, ReaL synthesis was induced with L-arabinose ( $0.2 \%$ final concentration), and the cells were cultured for another $30 \mathrm{~min}$. Then, the cells were harvested by centrifugation at $12,000 \mathrm{~g}$ for $5 \mathrm{~min}$ and total RNA was isolated as described above. Next, reverse transcription was performed with AMV reverse transcriptase (Promega), $1 \mu \mathrm{g}$ total RNA, and the rpoS specific primer Z144 (Supplementary Table S2). ReaL-rpoS chimera were amplified by PCR using primers Y144 and Z144 (Supplementary Table S2), and a $1-\mu 1$ aliquot of the reverse transcription reaction as template. The PCR products were purified using a Gel Extraction kit (QIAGEN) and cloned

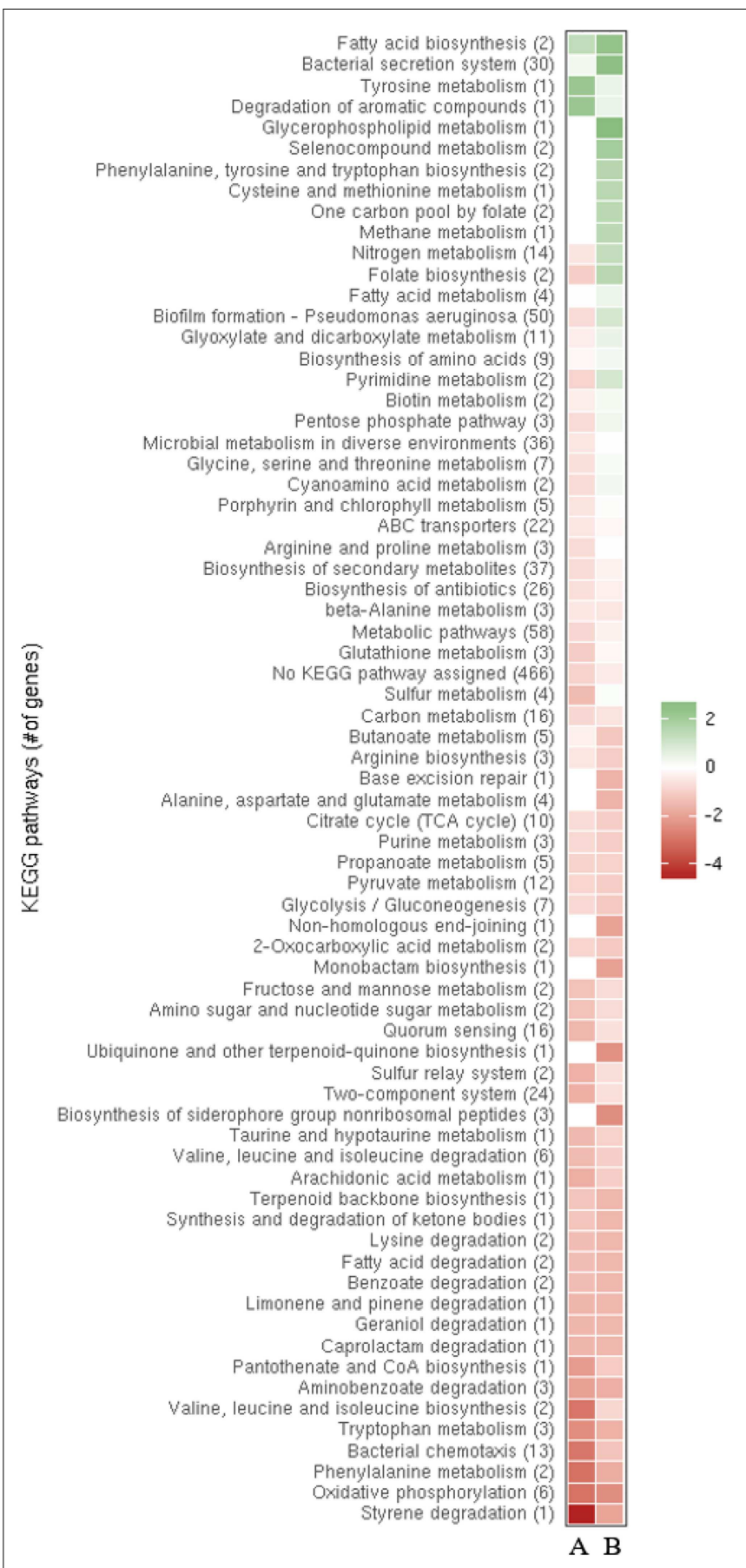

FIGURE 1 | Meta-analysis of normalized expression of differentially abundant transcripts in strains PAO1 $\Delta$ reaL(pKH6-ReaL) versus PAO1 1 reaL(pKH6) and strains PAO1DrpoS versus PAO1 (Schuster et al., 2004). The genes are grouped into the corresponding pathways

(http://www.kegg.jp/kegg-bin/show_organism?org = pau). The columns denote PAO1 1 reaL(pKH6-ReaL) versus PAO1 $\Delta$ reaL $(\mathrm{pKH} 6)$ (A) and PAO1 1 rpoS versus PAO1 (B), respectively. The color code shown in the scale at the right denotes $\log _{2}$-fold changes and fold change for the RNA $A_{S e q}$-based analysis, and the microarray-based analysis, respectively. Red indicates an overall decrease and green indicates an overall increase in the transcript levels of genes in a particular pathway. The numbers of genes within each pathway are indicated by the numbers given in parenthesis. 


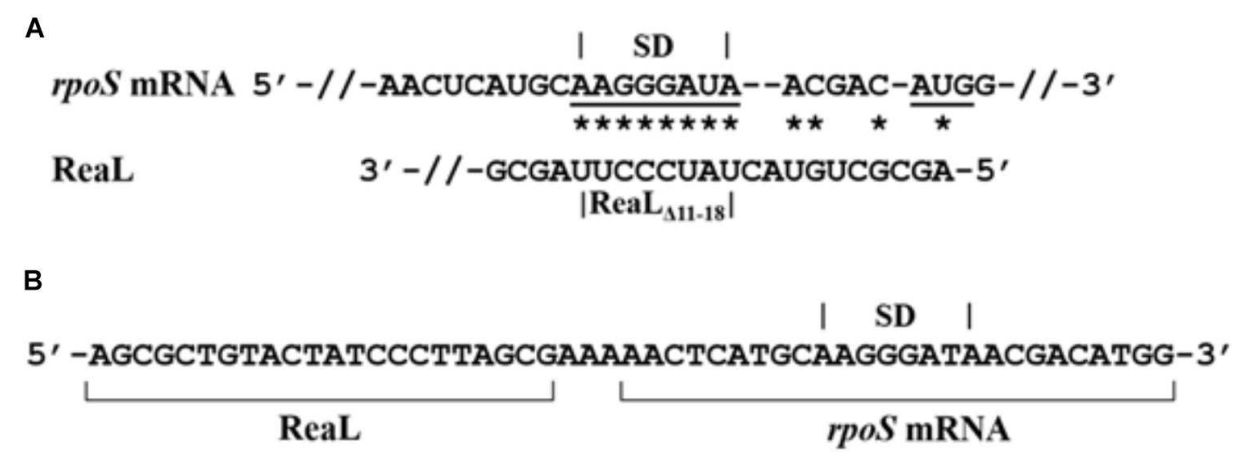

FIGURE 2 | Interaction of ReaL with the TIR of rpoS mRNA. (A) Complementarity of ReaL with the TIR of rpoS mRNA predicted with the IntaRNA algorithm (Mann et al., 2017). Possible base-pairing interactions are denoted by stars. The rpoS SD region and the start codon are underlined, respectively. The deletion of nt 11-18 present in ReaL $\mathrm{L}_{11-18}$ is indicated below. (B) ReaL-rpoS mRNA RLM-RT-PCR. The sub-sequence generated by ligation of ReaL with rpoS mRNA is shown. The ReaL and rpoS mRNA sequences are indicated by brackets and the rpoS SD region is denoted on top.

into the SmaI site of plasmid pUC19. Finally, the inserts were sequenced using the universal sequencing primer M13 (Supplementary Table S2).

\section{$\beta$-Galactosidase Assays}

Briefly, PAO1 $\Delta$ reaL(pKH6; pME6014-RpoS), PAO1 $\Delta$ reaL (pKH6-ReaL; pME6014-RpoS), PAO1 $\Delta$ reaL(pKH6-ReaL ${ }_{\Delta 11-18}$; pME6014-RpoS), PAO1 $\Delta r e a L(p K H 6 ;$ pME6016-RpoS), and PAO1 $\Delta$ reaL(pKH6-ReaL; pME6016-RpoS) were grown in triplicates in $20 \mathrm{ml} \mathrm{LB}$ broth. At an $\mathrm{OD}_{600}$ of 2.5, the synthesis of ReaL or of the $\operatorname{ReaL}_{\Delta 11-18}$ variant was induced with L-arabinose $(0.2 \%$ final concentration). Cells were cultured for another $30 \mathrm{~min}$ after which samples were withdrawn. Then, the $\beta$-galactosidase activities were determined from equal volumes of cells as described (Miller, 1972).

\section{RESULTS}

\section{Negative Regulation of RpoS Dependent Genes by ReaL}

To identify novel sRNAs in PAO1, an in silico search was performed using the general scheme described by Lenz et al. (2004). This screen revealed the putative sRNA PaeIII (SorgerDomenigg, 2010), which was later also identified with an RNA approach as SPA0084 (Ferrara et al., 2012), and recently renamed ReaL (Carloni et al., 2017). The expression of reaL in strain PAO1 was confirmed by Northern blot analysis (Supplementary Figure S1B). The transcriptional start site of reaL in sPAO1 was mapped to genome position 3958053 by a $5^{\prime}-3^{\prime}$ circularization approach (Supplementary Figure S1C). Based on this information, a PAO1 1 reaL strain was generated and transformed with either the reaL encoding plasmid pKH6-ReaL or the parental vector pKH6. As shown by Northern-blot analyses, ReaL was synthesized in PAO $\Delta$ reaL(pKH6-ReaL) and was absent in PAO1 $\Delta$ reaL(pKH6) (Supplementary Figure S2).

To study the impact of ReaL on global transcription, a comparative RNA $_{\text {Seq }}$ based transcriptome analysis was performed with PAO1 $\Delta$ reaL(pKH6-ReaL) and
PAO1 1 reaL(pKH6). A p-value (adjusted for multiple testing) of 0.05 was set as a threshold for significance and the change in abundance for a given transcript had to exceed a $\log _{2} \pm 1.5$-fold change to be considered differentially abundant (Supplementary Table S3). In brief, the largest differential abundance was observed for transcripts whose functions are involved in anaerobic respiration, in the synthesis of fimbriae, carbon source utilization, production of siderophores, and other virulence factors, many of which are regulated by RpoS (Schuster et al., 2004; Supplementary Table S3). In fact, the transcriptional profile of the PAO1 $\Delta$ reaL(pKH6-ReaL) strain resembled that previously described for a PAO $1 \Delta$ rpoS strain at the level of individual transcripts (Schuster et al., 2004). Next, a metaanalysis of normalized expression of differentially abundant transcripts was performed for the following strain pairs: PAO1 $\Delta$ reaL(pKH6-ReaL) versus PAO1 $\Delta$ reaL(pKH6) and PAO1 $1 r p o S$ versus PAO1. For this purpose, the genes were grouped into the corresponding KEGG pathways. ${ }^{2}$ As shown in the heat-map, the transcriptional profiles of the ReaL overproducing strain and the $r p o S$ deletion strain were closely related at the level of the KEGG pathways (Figure 1). Very similar changes in the abundance of transcripts assigned to distinct pathways were observed for PAO1 $\Delta$ reaL(pKH6-ReaL) and PAO $1 \Delta r p o S$ when compared with strains PAO1 $\Delta$ reaL(pKH6) and PAO1, respectively (Figure 1). In summary, these analyses suggested that ReaL might negatively impact RpoS synthesis.

\section{ReaL Interacts With rpoS mRNA}

As judged from the RNA Seq $_{\text {analysis, the transcript levels of } r p o S}$ were $\sim 19$-fold decreased in strain PAO1 $\Delta$ reaL(pKH6-ReaL) when compared with PAO1 $\Delta r e a L(\mathrm{pKH} 6)$ (Supplementary Table S3) 30 min after induction of the reaL gene, which could be a consequence of translational silencing of rpoS mRNA by ReaL followed by degradation of the mRNA. Similarly, qPCR revealed a $\sim 18$-fold decrease in the rpoS levels under the same experimental conditions (Supplementary Figure S3). In addition, the half-life of $r p o S$ mRNA was $\sim$ fivefold reduced in the ReaL overproducing

${ }^{2}$ http://www.kegg.jp/kegg-bin/show_organism?org=pau 
strain when compared with the control strain (Supplementary Figure S3). Therefore, an in silico approach (IntaRNA) (Mann et al., 2017) was first used to explore whether ReaL might interact with the translation initiation region (TIR) of $r p o S$ mRNA. This analysis suggested an interaction between the first $18 \mathrm{nt}$ of ReaL and the TIR of rpos (Figure 2A).

To corroborate the predicted ReaL-rpoS mRNA interaction in vivo, RNA ligase-mediated RT-PCR (RLM-RT-PCR) based on the GRIL-seq approach (Han et al., 2016) was employed. Briefly, GRIL-seq uses the ability of T4 RNA ligase to link two base-paired RNA molecules in vivo. In case of basepairing between a sRNA and its target RNA(s), chimeras of the sRNA and the target RNA(s) are generated. These chimeras are then reverse transcribed, amplified, and sequenced to identify targets for a given sRNA. The strains PAO1 $\Delta$ reaL(pKH6) and PAO1 $\Delta$ reaL(pKH6-ReaL) were additionally transformed with plasmid pKH13-t4rnl1, encoding the T4RNA ligase gene. ReaL and T4RNA ligase synthesis was induced with IPTG and L-arabinose, respectively. Then RLM-RT-PCR was performed with total RNA isolated from either strain, using rpoS and reaL specific oligonucleotides (Supplementary Table S2). A product of $200 \mathrm{nt}$ in length was detected in total RNA isolated from strain PAO1 $\Delta$ reaL(pKH6-ReaL; pKH13-t4rnl1), whereas this product was absent in total RNA isolated from strain PAO1 $\Delta$ reaL(pKH6; pKH13-t4rnl1). Cloning and sequencing of the 200 nt product detected in PAO1 $\Delta$ reaL(pKH6-ReaL; pKH13-t4rnl1) confirmed the formation of a ReaL-rpoS chimera in vivo (Figure 2B). This chimera contained the first $22 \mathrm{nt}$ of ReaL, an adenosine triplet followed by the sub-sequence of the rpoS mRNA predicted to interact with ReaL.

\section{ReaL Inhibits RpoS Synthesis}

As the modified GRIL-seq approach supported the hypothesis that ReaL inhibits rpos translation, we next assessed the steady-state levels of RpoS protein in strains PAO1 $\Delta r p o S$, PAO1 $\Delta$ reaL(pKH6), and PAO1 $\Delta$ reaL(pKH6-ReaL). These strains were cultured in LB until they reached an $\mathrm{OD}_{600}$ of 1 . Then, ReaL synthesis was induced with L-arabinose, and the cells were further grown for $30 \mathrm{~min}$. The RpoS levels were assessed through quantitative Western-blotting. A lower $\mathrm{OD}_{600}$ value for ReaL induction was chosen for this analysis to minimize detection of RpoS synthesized prior to ReaL induction. In contrast to strain PAO1 $\Delta$ reaL(pKH6), the RpoS levels were greatly diminished ( $>10$-fold) in the ReaL over-producing strain PAO1 $\Delta$ reaL (pKH6-ReaL) (Figure 3A).

To verify this finding, a translational rpoS::lacZ reporter gene, mounted on plasmid pME6014-RpoS, was employed. Strain PAO1 1 reaL(pME6014-RpoS) was transformed with plasmids pKH6, pKH6-ReaL, and pKH6-ReaL ${ }_{\Delta 11-18}$, respectively. Plasmid pKH6-ReaL $L_{\Delta 11-18}$ encodes a truncated variant of ReaL lacking nucleotides $11-18$, which are predicted to interact with rpoS mRNA (Figure 2A). The $\beta$-galactosidase activities conferred by plasmid pME6014-RpoS in strains PAO1 1 reaL(pKH6; pME6014-RpoS) and PAO1 1 reaL(pKH6ReaL; pME6014-RpoS) (Figure 3B) showed that reaL expression leads to a significant reduction of rpoS::lac $Z$ translation. However, expression of the rea $L_{\Delta 11-18}$ variant resulted

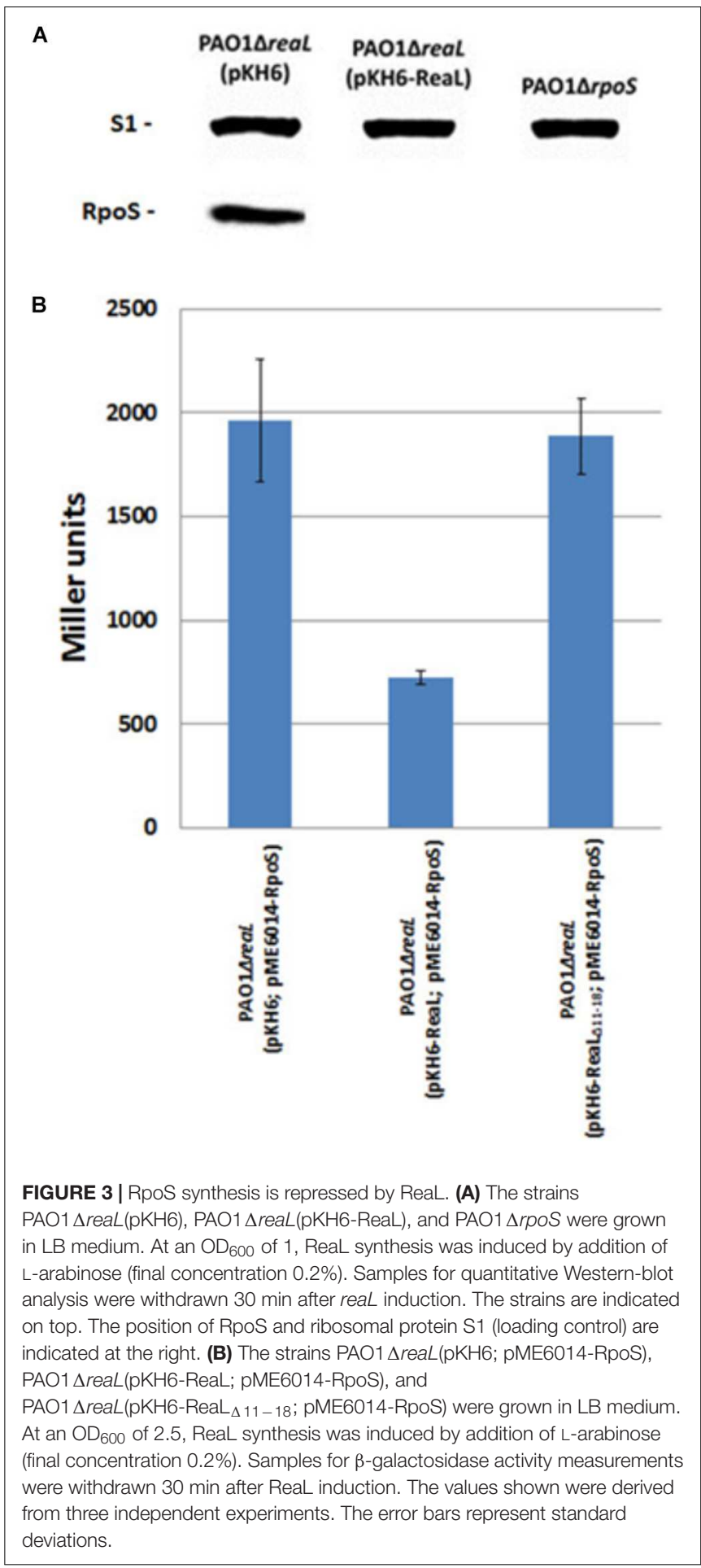

in $\beta$-galactosidase activities similar to those observed with the control strain PAO1 $\Delta$ reaL(pKH6; pME6014-RpoS). As the ReaL levels were similar in strains PAO1 $\Delta$ reaL(pKH6ReaL; pME6014-RpoS), and PAO1 $\Delta$ reaL(pKH6-ReaL $\mathrm{p}_{\Delta 11-18 ;}$ pME6014-RpoS) (Supplementary Figure S2), and as ReaL synthesis did not impact on the expression of a transcriptional rpoS-lacZ fusion gene (Supplementary Figure S4), we concluded 


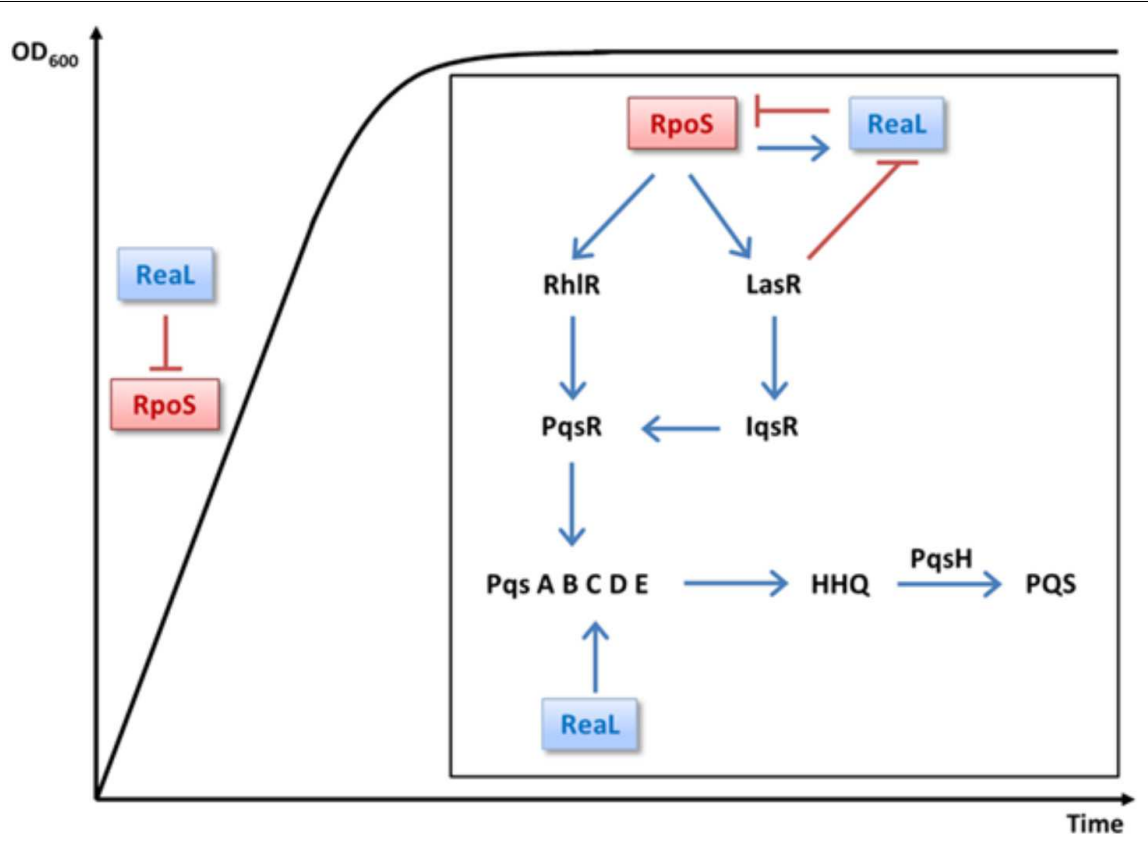

FIGURE 4 | Integration of ReaL in the multi-layered quorum sensing cascade. $\sigma^{70}$-dependent reaL transcription occurs during logarithmic growth (Sorger-Domenigg, 2010; Carloni et al., 2017). Continuous ReaL synthesis may prevent rpoS translation during logarithmic growth to avoid needless gene expression. When the cells encounter stationary phase, RpoS-mediated transcription of reaL increases through RpoS (Carloni et al., 2017), which in turn leads to repression of rpoS translation by a mixed negative feedback loop. When the cell density increases, LasR bound to its cognate autoinducer represses reaL transcription (Carloni et al., 2017), alleviating ReaL-mediated repression of RpoS synthesis, which in turn would stimulate LasR and RhIR synthesis (Schuster et al., 2004), and consequently the synthesis of PqsR and lqsR. Thus, in conjunction with other upstream regulators (Lee and Zhang, 2015), RpoS would act on top of a coherent feed-forward loop that serves to induce QS dependent genes. Positive and negative regulation is denoted by arrows and bars, respectively.

that the $5^{\prime}$-terminal sequence of ReaL is required for base-pairing with and to inhibit rpoS translation.

The study by Carloni et al. (2017) indicated that ReaL acts by base-pairing with $p q s C$ mRNA and that it positively stimulates translation of the $p q s C$ gene in a Hfq-independent manner. To test whether ReaL-mediated translational silencing of rpoS mRNA requires $\mathrm{Hfq}$, we compared the $\beta$-galactosidase activities conferred by plasmid pME6014-rpoS in strains PAO1 (pKH6-ReaL; pME6014-RpoS), PAO1(pKH6; pME6014-RpoS), PAO1 $\Delta h f q(\mathrm{pKH} 6-\mathrm{ReaL} ; \mathrm{pME6014-RpoS),}$ and PAO1 $\Delta h f q(\mathrm{pKH} 6$; pME6014-RpoS). In contrast to strain PAO1(pKH6-ReaL; pME6014-RpoS), rpoS translation did not decrease in the $h f q$ deletion strain (Supplementary Figure S5), suggesting that negative regulation of rpoS by ReaL is Hfq-dependent.

\section{DISCUSSION}

Here, we identified ReaL as the first known bacterial sRNA, which apparently represses rpoS translation by a base-pairing mechanism in a Hfq-dependent manner. The interaction between ReaL and rpoS mRNA is supported by the modified GRIL-seq approach (Figure 2B) and by the observation that ReaL $L_{D 11-18}$ was defective in repressing rpoS translation (Figure 3B). We attempted to obtain further evidence for the ReaL-rpoS mRNA interaction by introducing mutations into the TIR of rpoS mRNA.
However, compensatory mutations in the $5^{\prime}$ UTR of $r p o S$ could not be constructed because of their effect on translation initiation due to the disruption of the Shine-Dalgarno sequence; indeed, all attempted mutations affected translation of rpoS mRNA per se. This excluded the possibility to study ReaL-mediated regulation of $r p o S$ in more detail. In any case, ReaL overproduction reduced the rpoS transcript (Supplementary Table S3 and Figure S3) and protein levels (Figure 3A), respectively. It turn, translational silencing of rpoS mRNA resulted in faster degradation of the mRNA in the presence of ReaL (Supplementary Figure S3) as previously observed for RyhB-mediated translational repression of sodB mRNA (Massé and Gottesman, 2002; Afonyushkin et al., 2005).

By comparing the transcriptomes of PAO1 and a PAO1DrpoS strain, Schuster et al. (2004) reported that RpoS positively regulates the QS response regulator genes las $R$ and $r h l R \sim 2$ fold, which is in agreement with our $\mathrm{RNA}_{\mathrm{Seq}}$ analysis performed with the strains PAO1 $\Delta$ reaL(pKH6) and PAO1 $\Delta$ reaL(pKH6ReaL) (not shown). Hence, several differentially abundant transcripts/operons (Figure 1) and QS-mediated phenotypes reported upon over-production of ReaL (Carloni et al., 2017) might result from RpoS-mediated regulation of lasR/rhlR. When QS comes into effect the LasR regulator is the first to be activated, which in turn activates the Rhl, Pqs, and Iqs systems (Lee and Zhang, 2015). Thus, ReaL-mediated repression of $r p o S$, and consequently reduced synthesis of las $R$ would explain the diminished transcription of LasR-dependent genes/operons, 
such as the psl operon (Supplementary Table S3; Gilbert et al., 2009), encoding functions for matrix polysaccharide- or functions required for pyoverdine synthesis (Supplementary Table S3). The increase in pyocyanin production observed after ReaL overproduction (Carloni et al., 2017) is phenotypically in accord with the increased production of pyocyanin observed in a PAO1DrpoS strain (Suh et al., 1999; Whiteley et al., 2000; Diggle et al., 2002). However, ReaL-mediated over-production of pyocyanin was LasR/I independent (Carloni et al., 2017), and can therefore not be reconciled with its positive impact on LasR transcription (Schuster et al., 2004). ReaL-mediated stimulation of pyocyanin production was dependent on RhlR (Carloni et al., 2017), which can regulate PQS production in a positive (Dekimpe and Deziel, 2009) as well as in a negative manner (Brouwer et al., 2014). Given the complexity and interdependence of QS regulation more studies are required to test how ReaLmediated negative regulation of rpoS impacts on the Rhl system and how this affects pyocyanin production. The increase in pyocyanin synthesis upon ReaL overproduction has been mainly attributed to ReaL-mediated translational stimulation of the $p q s C$ gene, the function of which is required for production of the quinolone signal PQS (Figure 4; Carloni et al., 2017). However, as ReaL regulates several QS-dependent genes (Supplementary Table S3), which are likewise regulated by RpoS, its impact seems not to be restricted to PQS synthesis but it seems to operate as well in a hierarchical manner by acting on top of the cascade (Figure 4).

Despite the similarities in phenotypes and in the transcriptional profiles observed for the rpoS deletion mutant (Schuster et al., 2004) and the ReaL over-producing strain, a large number of genes displayed differential abundance in either strains (Supplementary Table S3). On one hand, these differences may arise from the different methods used for the transcriptome analysis, viz DNA microarrays (Schuster et al.,

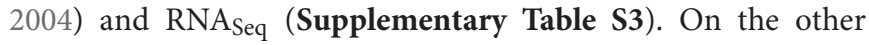
hand, it is possible that the regulatory role of ReaL extends beyond rpoS repression.

A working model, which integrates RpoS and ReaL in the multi-layered QS network is presented in Figure 4. $s^{70}$-dependent reaL transcription is observed during logarithmic growth and peaks in stationary phase (Sorger-Domenigg, 2010; Carloni et al., 2017). Therefore, continuous ReaL synthesis may prevent rpos translation during logarithmic growth to avoid superfluous gene expression. When the cells enter stationary phase, RpoSmediated transcription of reaL increases through stimulation

\section{REFERENCES}

Afonyushkin, T., Veèerek, B., Moll, I., Bläsi, U., and Kaberdin, V. R. (2005). Both RNase E and RNase III control the stability of sodB mRNA upon translational inhibition by the small regulatory RNA RyhB. Nucleic Acids Res. 33, 1678-1689. doi: 10.1093/nar/gki313

Bolger, A. M., Lohse, M., and Usadel, B. (2014). Trimmomatic: a flexible trimmer for Illumina sequence data. Bioinformatics 30, 2114-2120. doi: 10.1093/bioinformatics/btu170

Brouwer, S., Pustelny, C., Ritter, C., Klinkert, B., Narberhaus, F., and Häussler, S. (2014). The PqsR and RhlR transcriptional regulators determine the level of Pseudomonas Quinolone signal synthesis in Pseudomonas aeruginosa by RpoS, which in turn leads to repression of rpoS translation by a mixed negative feedback loop, as observed for RpoE and the sRNA RybB in E. coli (Thompson et al., 2007). When the cell density increases, LasR bound to its cognate autoinducer represses reaL transcription (Carloni et al., 2017), alleviating ReaL-mediated repression of RpoS synthesis, which in turn would stimulate LasR and RhlR synthesis (Schuster et al., 2004), and consequently the synthesis of PqsR and IqsR. Thus, in conjunction with other upstream regulators (Lee and Zhang, 2015), RpoS would act on top of a coherent feed-forward loop that serves to induce QS dependent genes.

\section{AUTHOR CONTRIBUTIONS}

UB and ES conceived and designed the experiments. HTBN, DRA, MT, and TS-D performed the experiments. UB, ES, HTBN, FA, and DRA analyzed the data. UB and DRA wrote the paper.

\section{FUNDING}

The work was supported by the Austrian Science Fund (www. fwf.ac.at/en) through the Special Research Program RNA-REG F43, subproject AF4311 (UB). DRA was supported by the FWF through a post-doctoral "Liese Meitner" fellowship (AM0206711). HTBN was supported by a "Ernst Mach" fellowship (ICM2017-06785) from the Austrian Federal Ministry of Science, Research, and Economy within the frame work of the ASEAUNINET program.

\section{ACKNOWLEDGMENTS}

We are grateful to Mr. Kook Han and Dr. Stephen Lory, Harvard Medical School, Boston, United States, for providing the materials for the GRIL-seq analysis and to Dr. Françoise Norel, Institut Pasteur, France, for providing the anti-RpoS antibodies.

\section{SUPPLEMENTARY MATERIAL}

The Supplementary Material for this article can be found online at: https://www.frontiersin.org/articles/10.3389/fmicb. 2018.02488/full\#supplementary-material

by producing two different pqsABCDE mRNA isoforms. J. Bacteriol. 196, 4163-4171. doi: 10.1128/JB.02000- 14

Caldelari, I., Chao, Y., Romby, P., and Vogel, J. (2013). RNA-mediated regulation in pathogenic bacteria. Cold Spring Harb. Perspect. Med. 3:a010298. doi: 10.1101/ cshperspect.a010298

Carloni, S., Macchi, R., Sattin, S., Ferrara, S., and Bertoni, G. (2017). The small RNA ReaL: a novel regulatory element embedded in the Pseudomonas aeruginosa quorum sensing networks. Environ. Microbiol. 19, 4220-4237. doi: 10.1111/ 1462-2920.13886

Chan, P. P., Holmes, A. D., Smith, A. M., Tran, D., and Lowe, T. M. (2012). The UCSC Archaeal Genome Browser: 2012 update. Nucleic Acids Res. 40, D646-D652. doi: 10.1093/nar/gkr990 
Cochran, W. L., Suh, S. J., Mcfeters, G. A., and Stewart, P. S. (2000). Role of RpoS and AlgT in Pseudomonas aeruginosa biofilm resistance to hydrogen peroxide and monochloramine. J. Appl. Microbiol. 88, 546-553. doi: 10.1046/j.1365-2672. 2000.00995.x

Costerton, J. W., Stewart, P. S., and Greenberg, E. P. (1999). Bacterial biofilms: a common cause of persistent infections. Science 284, 1318-1322. doi: 10.1126/ science.284.5418.1318

Dekimpe, V., and Deziel, E. (2009). Revisiting the quorum-sensing hierarchy in Pseudomonas aeruginosa: the transcriptional regulator RhlR regulates LasRspecific factors. Microbiology 155, 712-723. doi: 10.1099/mic.0.022764-0

Diggle, S. P., Winzer, K., Lazdunski, A., Williams, P., and Cámara, M. (2002). Advancing the Quorum in Pseudomonas aeruginosa: MvaT and the regulation of $\mathrm{N}$-acylhomoserine lactone production and virulence gene expression. J. Bacteriol. 184, 2576-2586. doi: 10.1128/JB.184.10.2576-2586.2002

Dong, T., and Schellhorn, H. E. (2010). Role of RpoS in virulence of pathogens. Infect. Immun. 78, 887-897. doi: 10.1128/IAI.00882-09

Ferrara, S., Brugnoli, M., De Bonis, A., Righetti, F., Delvillani, F., Dehò, G., et al. (2012). Comparative profiling of Pseudomonas aeruginosa strains reveals differential expression of novel unique and conserved small RNAs. PLoS One 7:e36553. doi: 10.1371/journal.pone.0036553

Ferrara, S., Carloni, S., Fulco, R., Falcone, M., Macchi, R., and Bertoni, G. (2015). Post-transcriptional regulation of the virulence-associated enzyme AlgC by the $\sigma 22$-dependent small RNA ErsA of Pseudomonas aeruginosa. Environ. Microbiol. 17, 199-214. doi: 10.1111/1462-2920.12590

Ferrara, S., Falcone, M., Macchi, R., Bragonzi, A., Girelli, D., Cariani, L., et al. (2017). The PAPI-1 pathogenicity island-encoded small RNA PesA influences Pseudomonas aeruginosa virulence and modulates pyocin S3 production. PLoS One 2:e0180386. doi: 10.1371/journal.pone.0180386

Fujita, M., Tanaka, K., Takahashi, H., and Amemura, A. (1994). Transcription of the principal sigma-factor genes, rpoD and rpoS, in Pseudomonas aeruginosa is controlled according to the growth phase. Mol. Microbiol. 13, 1071-1077. doi: 10.1111/j.1365-2958.1994.tb00498.x

Gilbert, K. B., Kim, T. H., Gupta, R., Greenberg, E. P., and Schuster, M. (2009). Global position analysis of the Pseudomonas aeruginosa quorum-sensing transcription factor LasR. Mol. Microbiol. 73, 1072-1085. doi: 10.1111/j.13652958.2009.06832.x

Gómez-Lozano, M., Marvig, R. L., Molin, S., and Long, K. S. (2012). Genomewide identification of novel small RNAs in Pseudomonas aeruginosa. Environ. Microbiol. 14, 2006-2016. doi: 10.1111/j.1462-2920.2012.02759.x

Han, K., Tjaden, B., and Lory, S. (2016). GRIL-seq provides a method for identifying direct targets of bacterial small regulatory RNA by in vivo proximity ligation. Nat. Microbiol. 2:16239. doi: 10.1038/nmicrobiol.2016.239

Hoe, C. H., Raabe, C. A., Rozhdestvensky, T. S., and Tang, T. H. (2013). Bacterial sRNAs: regulation in stress. Int. J. Med. Microbiol. 303, 217-229. doi: 10.1016/j. ijmm.2013.04.002

Hoffmann, S., Otto, C., Doose, G., Tanzer, A., Langenberger, D., Christ, S., et al. (2014). A multi-split mapping algorithm for circular RNA, splicing, transsplicing and fusion detection. Genome Biol. 15:R34. doi: 10.1186/gb-2014-152-r34

Hoffmann, S., Otto, C., Kurtz, S., Sharma, C. M., Khaitovich, P., Vogel, J., et al. (2009). Fast mapping of short sequences with mismatches, insertions and deletions using index structures. PLoS Comput. Biol. 5:e1000502. doi: 10.1371/ journal.pcbi.1000502

Hogardt, M., Roeder, M., Schreff, A. M., Eberl, L., and Heesemann, J. (2004). Expression of Pseudomonas aeruginosa exoS is controlled by quorum sensing and RpoS. Microbiology 150, 843-851. doi: 10.1099/mic.0.26703-0

Jorgensen, F., Bally, M., Chapon-Herve, V., Michel, G., Lazdunski, A., Williams, P., et al. (1999). RpoS-dependent stress tolerance in Pseudomonas aeruginosa. Microbiology 145(Pt 4), 835-844.

Lapouge, K., Schubert, M., Allain, F. H., and Haas, D. (2008). Gac/Rsm signal transduction pathway of $\gamma$-proteobacteria: from RNA recognition to regulation of social behaviour. Mol. Microbiol. 67, 241-253. doi: 10.1111/j.1365-2958.2007. 06042.x

Lee, J., and Zhang, L. (2015). The hierarchy quorum sensing network in Pseudomonas aeruginosa. Protein Cell 6, 26-41. doi: 10.1007/s13238-0140100-x

Lee, K. M., Go, J., Yoon, M. Y., Park, Y., Kim, S. C., Yong, D. E., et al. (2012). Vitamin B12-mediated restoration of defective anaerobic growth leads to reduced biofilm formation in Pseudomonas aeruginosa. Infect. Immun. 80, 1639-1649. doi: 10.1128/IAI.06161-11

Lenz, D. H., Mok, K. C., Lilley, B. N., Kulkarni, R. V., Wingreen, N. S., and Bassler, B. L. (2004). The small RNA chaperone Hfq and multiple small RNAs control quorum sensing in Vibrio harveyi and Vibrio cholerae. Cell 118, 69-82. doi: 10.1016/j.cell.2004.06.009

Leoni, L., Ciervo, A., Orsi, N., and Visca, P. (1996). Iron-regulated transcription of the pvdA gene in Pseudomonas aeruginosa: effect of Fur and PvdS on promoter activity. J. Bacteriol. 178, 2299-2313. doi: 10.1128/jb.178.8.2299-2313. 1996

Livny, J., Brencic, A., Lory, S., and Waldor, M. K. (2006). Identification of 17 Pseudomonas aeruginosa sRNAs and prediction of sRNA-encoding genes in 10 diverse pathogens using the bioinformatic tool sRNAPredict2. Nucleic Acids Res. 34, 3484-3493. doi: 10.1093/nar/gkl453

Love, M. I., Huber, W., and Anders, S. (2014). Moderated estimation of fold change and dispersion for RNA-seq data with DESeq2. Genome Biol. 15:550. doi: 10.1186/s13059-014-0550-8

Lu, P., Wang, Y., Hu, Y., and Chen, S. (2018). RgsA, an RpoS-dependent sRNA, negatively regulates rpoS expression in Pseudomonas aeruginosa. Microbiology 164, 716-724. doi: 10.1099/mic.0.000632

Majdalani, N., Cunning, C., Sledjeski, D., Elliott, T., and Gottesman, S. (1998). DsrA RNA regulates translation of rpoS message by an anti-antisense mechanism, independent of its action as an antisilencer of transcription. Proc. Natl. Acad. Sci. U.S.A. 95, 12462-12467. doi: 10.1073/pnas.95.21.12462

Majdalani, N., Hernandez, D., and Gottesman, S. (2002). Regulation and mode of action of the second small RNA activator of RpoS translation, RprA. Mol. Microbiol. 46, 813-826. doi: 10.1046/j.1365-2958.2002.03203.x

Mann, M., Wright, P. R., and Backofen, R. (2017). IntaRNA 2.0: enhanced and customizable prediction of RNA-RNA interactions. Nucleic Acids Res. 45, W435-W439. doi: 10.1093/nar/gkx279

Martínez-Bueno, M. A., Tobes, R., Rey, M., and Ramos, J. L. (2002). Detection of multiple extracytoplasmic function (ECF) sigma factors in the genome of Pseudomonas putida KT2440 and their counterparts in Pseudomonas aeruginosa PA01. Environ. Microbiol. 4, 842-855. doi: 10.1046/j.1462-2920. 2002.00371.x

Massé, E., and Gottesman, S. (2002). A small RNA regulates the expression of genes involved in iron metabolism in Escherichia coli. Proc. Natl. Acad. Sci. U.S.A. 99, 4620-4625. doi: 10.1073/pnas.032066599

McCullen, C. A., Benhammou, J. N., Majdalani, N., and Gottesman, S. (2010). Mechanism of positive regulation by DsrA and RprA small noncoding RNAs: pairing increases translation and protects rpoS mRNA from degradation. J. Bacteriol. 192, 5559-5571. doi: 10.1128/JB.00464-10

Mika, F., and Hengge, R. (2014). Small RNAs in the control of RpoS, CsgD, and biofilm architecture of Escherichia coli. RNA Biol. 11, 494-507. doi: 10.4161/ rna.28867

Miller, C. L., Romero, M., Karna, S. L. R., Chen, T., Heeb, S., and Leung, K. P. (2016). RsmW, Pseudomonas aeruginosa small non-coding RsmA-binding RNA upregulated in biofilm versus planktonic growth conditions. BMC Microbiol. 16:155. doi: 10.1186/s12866-016-0771-y

Miller, J. H. (1972). Experiments in Molecular Genetics. Cold Spring Harbor, NY: Cold Spring Harbor Laboratory.

Pfaffl, M. W. (2001). A new mathematical model for relative quantification in real-time RT-PCR. Nucleic Acids Res. 29:e45. doi: 10.1093/nar/29.9.e45

Quinlan, A. R., and Hall, I. M. (2010). BEDTools: a flexible suite of utilities for comparing genomic features. Bioinformatics 26, 841-842. doi: 10.1093/ bioinformatics/btq033

Reinhart, A. A., Powell, D. A., Nguyen, A. T., O’neill, M., Djapgne, L., Wilks, A., et al. (2015). The prrF-encoded small regulatory RNAs are required for iron homeostasis and virulence of Pseudomonas aeruginosa. Infect. Immun. 83, 863-875. doi: 10.1128/IAI.02707-14

Schuster, M., Hawkins, A. C., Harwood, C. S., and Greenberg, E. P. (2004). The Pseudomonas aeruginosa RpoS regulon and its relationship to quorum sensing. Mol. Microbiol. 51, 973-985. doi: 10.1046/j.1365-2958.2003.03886.x

Sonnleitner, E., and Bläsi, U. (2014). Regulation of Hfq by the RNA CrcZ in Pseudomonas aeruginosa carbon catabolite repression. PLoS Genet. 10:e1004440. doi: 10.1371/journal.pgen.1004440

Sonnleitner, E., Gonzalez, N., Sorger-Domenigg, T., Heeb, S., Andreas, S., Richter, A. S., et al. (2011). The small RNA PhrS stimulates synthesis of the Pseudomonas 
aeruginosa quinolone signal. Mol. Microbiol. 80, 868-885. doi: 10.1111/j.13652958.2011.07620.x

Sonnleitner, E., and Haas, D. (2011). Small RNAs as regulators of primary and secondary metabolism in Pseudomonas species. Appl. Microbiol. Biotechnol. 91, 63-79. doi: 10.1007/s00253-011-3332-1

Sonnleitner, E., Hagens, S., Rosenau, F., Wilhelm, S., Habel, A., Jager, K. E., et al. (2003). Reduced virulence of a hfq mutant of Pseudomonas aeruginosa O1. Microb. Pathog. 35, 217-228. doi: 10.1016/S0882-4010(03)00149-9

Sonnleitner, E., Sorger-Domenigg, T., Madej, M. J., Findeiss, S., Hackermüller, J., Hüttenhofer, A., et al. (2008). Detection of small RNAs in Pseudomonas aeruginosa by RNomics and structure-based bioinformatic tools. Microbiology 154, 3175-3187. doi: 10.1099/mic.0.2008/019703-0

Sorger-Domenigg, T. (2010). Novel Pseudomonas Aeruginosa Small Regulatory RNAs. PhD thesis, University of Vienna, Vienna.

Storz, G., Vogel, J., and Wassarman, K. M. (2011). Regulation by small RNAs in Bacteria: expanding frontiers. Mol. Cell 43, 880-891. doi: 10.1016/j.molcel.2011. 08.022

Suh, S. J., Silo-Suh, L., Woods, D. E., Hassett, D. J., West, S. E., and Ohman, D. E. (1999). Effect of rpoS mutation on the stress response and expression of virulence factors in Pseudomonas aeruginosa. J. Bacteriol. 181, 3890-3897.

Thompson, K. M., Rhodius, V. A., and Gottesman, S. (2007). $\sigma(\mathrm{E})$ regulates and is regulated by a small RNA in Escherichia coli. J. Bacteriol. 189, 4243-4256. doi: 10.1128/JB.00020-07

Toledo-Arana, A., Dussurget, O., Nikitas, G., Sesto, N., Guet-Revillet, H., Balestrino, D., et al. (2009). The Listeria transcriptional landscape from saprophytism to virulence. Nature 459, 950-956. doi: 10.1038/nature08080

Van Delden, C., and Iglewski, B. H. (1998). Cell-to-cell signaling and Pseudomonas aeruginosa infections. Emerg. Infect. Dis. 4, 551-560.

Venturi, V. (2003). Control of rpoS transcription in Escherichia coli and Pseudomonas: why so different? Mol. Microbiol. 49, 1-9.

Wenner, N., Maes, A., Cotado-Sampayo, M., and Lapouge, K. (2014). NrsZ: a novel, processed, nitrogen-dependent, small non-coding RNA that regulates Pseudomonas aeruginosa PAO1 virulence. Environ. Microbiol. 16, 1053-1068. doi: $10.1111 / 1462-2920.12272$
Whiteley, M., Parsek, M. R., and Greenberg, E. P. (2000). Regulation of quorum sensing by RpoS in Pseudomonas aeruginosa. J. Bacteriol. 182, 4356-4560. doi: 10.1128/JB.182.15.4356-4360.2000

Wolfinger, M. T., Fallmann, J., Eggenhofer, F., and Amman, F. (2015). ViennaNGS: a toolbox for building efficient next-generation sequencing analysis pipelines. F1000Res. 4:50. doi: 10.12688/f1000research.6157.2

Wosten, M. M. (1998). Eubacterial sigma-factors. FEMS Microbiol. Rev. 22, 127-150. doi: 10.1111/j.1574-6976.1998.tb00364.x

Wurtzel, O., Yoder-Himes, D. R., Han, K., Dandekar, A. A., Edelheit, S., Greenberg, E. P., et al. (2012). The single-nucleotide resolution transcriptome of Pseudomonas aeruginosa grown in body temperature. PLoS Pathog. 8:e1002945. doi: 10.1371/journal.ppat.1002945

Ye, R. W., Haas, D., Ka, J. O., Krishnapillai, V., Zimmermann, A., Baird, C., et al. (1995). Anaerobic activation of the entire denitrification pathway in Pseudomonas aeruginosa requires Anr, an analog of Fnr. J. Bacteriol. 177, 3606-3609. doi: 10.1128/jb.177.12.3606-3609.1995

Zhang, A., Altuvia, S., Tiwari, A., Argaman, L., Hengge-Aronis, R., and Storz, G. (1998). The OxyS regulatory RNA represses rpoS translation and binds the Hfq (HF-I) protein. EMBO J. 17, 6061-6068. doi: 10.1093/emboj/17.20.6061

Zhang, Y. F., Han, K., Chandler, C. E., Tjaden, B., Ernst, R. K., and Lory, S. (2017). Probing the sRNA regulatory landscape of $P$. aeruginosa: post-transcriptional control of determinants of pathogenicity and antibiotic susceptibility. Mol. Microbiol. 106, 919-937. doi: 10.1111/mmi.13857

Conflict of Interest Statement: The authors declare that the research was conducted in the absence of any commercial or financial relationships that could be construed as a potential conflict of interest.

Copyright (C) 2018 Thi Bach Nguyen, Romero A, Amman, Sorger-Domenigg, Tata, Sonnleitner and Bläsi. This is an open-access article distributed under the terms of the Creative Commons Attribution License (CC BY). The use, distribution or reproduction in other forums is permitted, provided the original author(s) and the copyright owner(s) are credited and that the original publication in this journal is cited, in accordance with accepted academic practice. No use, distribution or reproduction is permitted which does not comply with these terms. 